International Journal of Modern Physics D

Vol. 25, No. 3 (2016) 1692001 (1 page)

(C) World Scientific Publishing Company

DOI: $10.1142 / \mathrm{S} 0218271816920012$

\title{
Erratum
}

\section{Is gravitino condensation possible?}

\author{
[International Journal of Modern Physics D, Vol. 13, No. 5 (2004) 923-933] \\ M. D. Pollock \\ V. A. Steklov Mathematical Institute, \\ Russian Academy of Sciences, \\ Ulitsa Gubkina 8, Moscow 119991, Russia \\ mdp30@cam.ac.uk
}

Received 22 January 2016

Published 26 February 2016

The following are corrections to errors in the published paper

(1) On page 928, in Eq. (19), printed as

$$
\frac{1}{4} e^{-\sqrt{2} \kappa_{0} \theta|W|}
$$

should read

$$
\frac{1}{4} e^{-\sqrt{2} \kappa_{0} \theta}|W|
$$

(2) On page 932, Eq. (41) should read

$$
A_{\mathrm{r}}=\mathcal{K}^{2} / \mathcal{H}^{2} B_{\mathrm{r}}^{3}
$$

